\title{
Efeitos de Intervenções em Letramento Emergente: Uma Revisão Bibliográfica na Base SciELO*
}

\author{
Sylvia Domingos Barrera ${ }^{1}$, Iolanda Ribeiro ${ }^{2, * *}, \&$ Fernanda Leopoldina Viana ${ }^{2}$ \\ ${ }^{1}$ Universidade de São Paulo, Ribeirão Preto, SP, Brasil \\ ${ }^{2}$ Universidade do Minho, Minho, Portugal
}

\begin{abstract}
RESUMO - Na perspectiva do letramento emergente, a alfabetização é influenciada pelas seguintes competências: processamento fonológico, conhecimentos sobre a escrita e linguagem oral. Esta pesquisa teve como objetivo realizar um levantamento sistemático, na base de dados SciELO, de estudos que analisaram o efeito de programas visando ao desenvolvimento de habilidades de letramento emergente em pré-escolares nos últimos dez anos. Dos 15 estudos identificados, oito foram intervenções focadas no desenvolvimento da consciência fonológica, quatro tiveram foco na linguagem oral, baseando-se na leitura compartilhada e três abordaram ambos os tipos de habilidades. Apesar da diversidade, apenas um estudo não obteve efeitos positivos significativos. Os resultados sugerem maior eficácia de programas abordando tanto habilidades linguísticas, quanto metalinguísticas. Questões metodológicas e implicações educacionais são discutidas. PALAVRAS-CHAVE: letramento emergente, programas, consciência fonológica, leitura compartilhada, linguagem oral
\end{abstract}

\section{Effects of Emergent Literacy Interventions: A Literature Review in the Scielo Database}

\begin{abstract}
In the early literacy approach, learning to read is affected by phonological processing, print awareness and oral language skills. This study aimed to conduct a search in the SciELO database for research that analyzed the effect of programs seeking to develop early literacy skills in kindergartners in the past ten years. Fifteen studies were identified: eight were interventions focused on phonological awareness development, four were focused on oral language based on shared reading and three were focused on both sets of skills. Despite the diversity, only one study did not show significant effects. The results suggest that programs that integrate both linguistic and metalinguistic skills are more effective. Methodological questions and educational implications of the studies are also discussed.
\end{abstract}

KEYWORDS: early literacy, programs, phonological awareness, shared reading, oral language

Nos últimos 30 anos, crescente atenção vem sendo dada aos anos pré-escolares, como um período crítico para o desenvolvimento de habilidades relacionadas ao sucesso na aprendizagem da leitura e da escrita (Dickinson, McCabe, \& Essex, 2006; Wasik, Bond, \& Hindman, 2006). Resultados de pesquisas na área do letramento emergente, definido como o conjunto de conhecimentos, competências e atitudes consideradas precursoras da aprendizagem da leitura, abarcando igualmente os contextos que facilitam essa aprendizagem (Whitehurst \& Lonigan, 1998), levaram a mudanças no conceito de prontidão para alfabetização, o qual deixou de ser considerado como um processo essencialmente maturacional. Atualmente, existe um amplo consenso de que, nas sociedades letradas, as aprendizagens a respeito da língua escrita começam praticamente desde o nascimento, atingindo diferentes níveis de desenvolvimento antes mesmo do ingresso da criança no ensino formal. É também amplamente reconhecido que a qualidade da educação pré-escolar desempenha um papel fundamental no sucesso posterior das crianças como futuros leitores e escritores (Campos et al., 2011; Neuman \& Dickinson, 2003).

Estudos sugerem que crianças que iniciam o ensino formal com habilidades de letramento emergente bem

\footnotetext{
$\bar{*}$ Apoio FAPESP

** E-mail: sdbarrera@ffclrp.usp.br

- Submetido: 03/08/2015; Aceito: 23/03/2016.
} 
desenvolvidas encontram-se melhor preparadas para iniciar com sucesso a alfabetização (Storch \& Whitehurst, 2002; Whitehurst \& Lonigan, 1998). Segundo Whitehurst e Lonigan (2003), a aprendizagem da leitura é influenciada pelas seguintes competências de base: processamento fonológico, conhecimentos a respeito da escrita e linguagem oral.

De acordo com Wagner e Torgesen (1987), o processamento fonológico inclui a memória fonológica de curto prazo, o acesso ao léxico fonológico na memória de longo prazo e a consciência fonológica (CF), habilidade essa que tem sido muito pesquisada em suas relações com a aprendizagem da leitura e escrita. Bryant e Bradley (1987) definiram CF como a habilidade de reflexão sobre os sons que compõem a fala. Essa reflexão pode incidir sobre diferentes unidades linguísticas: palavras, sílabas, unidades intrassilábicas - como onset e rima - e fonemas, podendo também variar quanto ao grau de consciência ou controle intencional, conforme a distinção entre habilidades epilinguísticas (conhecimentos implícitos) versus habilidades metalinguísticas (conhecimentos explícitos) (Gombert, 2003).

Tais ambiguidades, na definição do constructo $\mathrm{CF}$, têm implicações importantes sobre a análise de suas relações com a aprendizagem da leitura. Assim, embora haja fortes evidências de que treinos em CF têm impacto significativo sobre a aprendizagem da leitura e escrita, sobretudo quando associados ao ensino das correspondências letra-som (Bus \& van IJzendoorn, 1999; Capovilla \& Capovilla, 2000; Ellis, 2001; Hatcher, Hulme, \& Ellis, 1994), autores como Melby-Lervag, Lyster e Hulme (2012) sugerem que apenas a consciência fonêmica desempenha papel preditivo específico e substancial para a aprendizagem da leitura.

Entre os conhecimentos sobre a escrita considerados relevantes para a sua aprendizagem formal, estão algumas convenções relacionadas ao texto (direção da escrita, separação entre palavras, pontuação), além de conhecimentos sobre as funções da escrita. Assim, tem sido enfatizada a importância de garantir aos pré-escolares o contato com vários tipos de materiais escritos, bem como a participação em práticas de leitura e escrita mediadas por adultos, tanto na pré-escola, quanto no lar (Justice, McGinty, Piasta, Kaderavek, \& Fan, 2010; Levin \& Aram, 2010).

Ainda no domínio dos conhecimentos sobre a escrita, um dos aspectos mais investigados tem sido o relativo aos conhecimentos sobre o alfabeto. De acordo com Schatschneider, Fletcher, Francis, Carlson e Foorman (2004), o conhecimento dos nomes e sons das letras é preditor da alfabetização. Pesquisas indicam que pré-escolares com pobre domínio dos nomes e sons das letras têm maior risco de apresentar dificuldades na aprendizagem da leitura e escrita (Torgesen, 2002; Cardoso-Martins \& Baptista, 2005). Entretanto, um estudo de meta-análise realizado por Piasta e Wagner (2010) encontrou mínima evidência dos efeitos da instrução do alfabeto sobre as habilidades de CF e escrita. Em leitura, foram encontrados pequenos efeitos dessa intervenção, porém esses não tendem a se manter a longo prazo. Contudo, os autores advertem para a necessidade de analisar com cautela tais resultados, devido à grande variabilidade das pesquisas quanto ao conteúdo e à metodologia, além de muitos estudos terem sido realizados com amostras pequenas.

Por fim, têm sido encontradas correlações e continuidade entre as habilidades de linguagem oral e leitura (Lonigan, Burgess, \& Anthony, 2000). Estudos sugerem que o vocabulário no início do ensino formal tem efeito preditor sobre a leitura de palavras ao final do primeiro ano, bem como sobre a compreensão em leitura em anos posteriores (Juel, 2006). Segundo Dickinson et al. (2006), a linguagem, entendida de forma ampla - incluindo vocabulário, sintaxe, discurso e consciência fonêmica - , pode ser considerada um aspecto central para o letramento inicial e mesmo para o sucesso futuro na leitura e escrita.

A meta-análise realizada por Mol, Bus, de Jong e Smeets (2008), analisando pesquisas sobre leitura compartilhada por pais treinados para utilizar estratégias dialógicas, concluiu que essa abordagem apresenta efeitos moderados sobre o vocabulário expressivo, sobretudo no caso de crianças menores (2 a 4 anos). Os efeitos sobre crianças mais velhas e sobre o vocabulário receptivo mostraram-se mais fracos, sendo também menores em crianças cujas mães tinham menor nível educacional. Lever e Sénéchal (2011) e Wasik et al. (2006) obtiveram efeitos positivos da leitura compartilhada e dialógica sobre a estruturação das narrativas orais e sobre o vocabulário de pré-escolares, quando realizada por pesquisadores e para pequenos grupos.

Vários pesquisadores têm estudado o efeito de programas de intervenção com pré-escolares, visando ao desenvolvimento das habilidades de letramento emergente. Shanahan e Lonigan (2010) relatam os principais resultados do The National Early Literacy Panel (NELP), estudo de metanálise publicado em 2008 que, a partir da análise de cerca de 500 artigos científicos, buscou identificar quais habilidades e competências melhor predizem o desempenho posterior na aprendizagem da leitura e da escrita e que abordagens instrucionais e ambientes educacionais mais contribuem para o desenvolvimento dessas habilidades.

Para responder a essa segunda questão, o painel analisou cerca de 190 estudos, descrevendo o impacto de vários tipos de instrução e programas no desenvolvimento das habilidades de letramento emergente. Os resultados demonstraram que intervenções focadas no código tiveram efeitos positivos significativos, de grau moderado a forte, sobre o conhecimento do alfabeto e das habilidades de CF, leitura e escrita, enquanto intervenções com base em leitura compartilhada tiveram impacto positivo moderado sobre as habilidades linguísticas das crianças, em especial sobre o vocabulário.

Tendo em vista que o NELP analisou, basicamente, pesquisas sobre letramento emergente produzidas em língua 
inglesa, considera-se relevante realizar um levantamento sobre o tema abordando da literatura produzida em português e em espanhol, uma vez que estudiosos da alfabetização vêm enfatizando as diferenças encontradas entre os processos cognitivos envolvidos na leitura e escrita em línguas com diferentes graus de regularidade nas correspondências grafofonológicas (Capovilla, Capovilla, \& Machalous, 2005).

Nesse sentido, considerando a representatividade da base de dados SciELO (Scientific Eletronic Library Online) no contexto ibero-americano, o presente estudo teve como objetivo geral realizar um levantamento sistemático, no banco de dados SciELO, de estudos que analisaram o efeito de programas e/ou intervenções com pré-escolares, voltados para o desenvolvimento de habilidades de letramento emergente, publicados nos últimos 10 anos. Como objetivos específicos, pretendeu-se, a partir da identificação da produção científica ibero-americana sobre o tema na última década, analisar a efetividade dos programas, bem como as características desses capazes de promover melhores resultados, além de discutir questões metodológicas e implicações pedagógicas dos resultados obtidos.

\section{MÉTODO}

A pesquisa foi realizada a partir de uma consulta sistemática à base de dados SciELO, utilizando-se os seguintes termos (descritores): emergent literacy/early literacy/letramento emergente/literacia emergente/ alfabetización temprana. Também foram realizadas buscas a partir das palavras program/ programa e intervention/ intervenção/ intervención, associadas a: kindergartners/ pré-escolares/preescolares; literacy/ alfabetização/ alfabetización; reading / leitura/ lectura; spelling/ escrita. Foram adotados os seguintes critérios para a inclusão dos artigos: estudos abordando o efeito de intervenções, tendo como objetivo desenvolver habilidades de letramento emergente em pré-escolares, publicados entre 2004 e 2014. Como critério de exclusão, não foram considerados artigos que abordavam intervenções realizadas com crianças com algum tipo de deficiência ou dificuldade específica de aprendizagem.
Os artigos identificados foram agrupados em três categorias, em função das principais habilidades trabalhadas: 1) programas focados no domínio do sistema de escrita alfabético (consciência fonológica, conhecimento das letras, relações grafema/fonema); 2) programas focados no desenvolvimento da linguagem oral (vocabulário, expressão e compreensão oral); 3) programas mistos, ou seja, que abordaram ambos os tipos de habilidades. Não foi julgada pertinente a realização de uma meta-análise pelo fato dos estudos exibirem diferenças importantes nas habilidades desenvolvidas pelos programas, nas medidas de resultado utilizadas e nas metodologias empregadas (cf. Rosenshine, 2001). De fato, tal diversidade pode ser atribuída, ao menos em parte, ao próprio constructo de "letramento emergente", o qual envolve competências cognitivas e linguísticas bastante diversificadas.

\section{RESULTADOS}

Entre 2004 e 2014, foram encontrados, na base de dados SciELO, 15 artigos abordando programas de intervenção relacionados às habilidades de letramento emergente. Desses, cinco foram publicados no Brasil, três na Argentina e três no Chile, sendo que Colômbia, Espanha, Portugal e Venezuela também aparecem representados, com um artigo publicado. Observa-se, ainda, que a maioria dos artigos (80\%) foi publicada a partir do ano de 2009.

Os participantes foram alunos de pré-escolas públicas, de nível socioeconômico (NSE) baixo, com idades entre 4 e 6 anos. Três pesquisas trabalharam com participantes em situação de risco, para apresentar dificuldades na aprendizagem da leitura e da escrita, devido ao desempenho abaixo do esperado em habilidades avaliadas no início dos estudos (Bizama, Arancibia, \& Sáez, 2013; Flórez-Romero, Restrepo, \& Schwanenflugel, 2009; Valenzuela, Ruiz, \& Ríos, 2011).

Entretanto, nas demais pesquisas, geralmente foi assumido, de forma mais ou menos explícita que, por se tratarem de crianças de NSE baixo, as mesmas se encontravam em risco de apresentar dificuldades na alfabetização, tendo em vista sua situação socioeconômica de vulnerabilidade. Entre os estudos analisados, oito (53\%) foram classificados como intervenções focadas em habilidades relacionadas à compreensão do sistema alfabético, quatro $(27 \%)$ tiveram como foco o desenvolvimento da linguagem oral e três $(20 \%)$ abordaram ambos os tipos de habilidades (programas mistos),

\section{Programas com Ênfase na Compreensão do Sistema Alfabético e na CF}

Das oito intervenções focadas na compreensão do sistema alfabético (Tabela 1), cinco dizem respeito a programas voltados especificamente para o desenvolvimento da $\mathrm{CF}$, sendo que os demais programas desse grupo também abordaram, de forma mais ou menos intensiva, essa habilidade metalinguística. Arancibia, Bizama e Sáez 
(2012) avaliaram os efeitos da intervenção sobre as próprias habilidades de CF. O estudo foi realizado com 20 préescolares, utilizando delineamento pré-teste/intervenção/ pós-teste, sem grupo-controle. Como medida de resultado, foi usada a mesma prova padronizada de segmentação linguística utilizada no pré-teste.

As pesquisadoras realizaram 15 sessões de 30 minutos, duas vezes por semana, em sala de aula, buscando desenvolver habilidades de análise da língua oral nos níveis lexical, silábico e fonêmico, com ênfase nos dois primeiros, tendo obtido ganhos significativos dos pré-escolares nas habilidades silábicas epilinguísticas. Já o estudo de Bizama, Arancibia e Sáez (2013) foi realizado com 41 pré-escolares em situação de risco, por habilidades iniciais reduzidas em CF e memória fonológica. A pesquisa seguiu um delineamento pré-teste, intervenção, pós-teste com Grupo Controle e utilizou o mesmo programa que o estudo anterior, aplicado nas mesmas condições, tendo obtido resultados significativos positivos a favor do Grupo Experimental no Pós-teste, tanto em CF, quanto na memória fonológica.

Outros três estudos (Dambrowski, Martins, Theodoro, \& Gomes, 2008; Pestun, Omote, Barreto, \& Matsuo, 2010; Santos \& Maluf, 2010) analisaram os efeitos da intervenção em CF sobre as habilidades metafonológicas e de escrita. Os programas de Dambrowski et al. (2008) e de Pestun et al. (2010) foram aplicados pelas pesquisadoras, utilizando delineamento experimental com grupo controle e, como medidas de resultado, o teste CONFIAS (Moojen, 2003, citado por Pestun et al., 2010), prova de CF padronizada, além de tarefas não padronizadas de escrita.

O estudo de Dambrowski et al. (2008), realizado com 56 pré-escolares de 5 a 6 anos, constou de 10 sessões semanais, com 30 minutos de duração, envolvendo, além de atividades voltadas para o desenvolvimento da $\mathrm{CF}$, treino em correspondências grafema-fonema, tendo obtido resultados positivos a favor do Grupo Experimental, tanto em CF, quanto em escrita. Já a pesquisa realizada por Pestun et al. (2010), com uma amostra de 88 crianças com idade média de 5 a 6 anos, embora prevista para ocorrer em 35 sessões de uma hora, acabou sendo desenvolvida em apenas 26 sessões, não havendo tempo para serem realizadas as atividades visando ao desenvolvimento da consciência fonêmica.

Os resultados obtidos não mostraram diferenças significativas entre Grupo Experimental e Grupo Controle. A pesquisa de Santos e Maluf (2010), realizada com uma amostra de 90 pré-escolares de 5 a 6 anos, utilizou delineamento quase experimental com grupo-controle. As autoras buscaram analisar, também, os efeitos do aplicador sobre os resultados do programa, a partir da variação de três condições de aplicação: pela própria pesquisadora, pela professora da turma ou pela coordenadora pedagógica da escola. Como medidas de resultados, foram utilizadas tarefas não padronizadas de $\mathrm{CF}$ e de escrita. $\mathrm{O}$ programa constou em 32 sessões, visando ao desenvolvimento da $\mathrm{CF}$, tendo sido obtidos resultados significativos a favor dos
Grupos Experimentais, tanto em CF, quanto em escrita, não tendo sido encontradas diferenças significativas em função do aplicador.

Dois estudos classificados nessa categoria consistiram em intervenções mais intensas e prolongadas, direcionadas a crianças consideradas em risco para apresentar dificuldades na alfabetização. A pesquisa de Ghiglione, Filipetti, Manucci e Apaz (2011) testou os efeitos de um programa adaptado ao currículo escolar sobre habilidades linguísticas (CF) e cognitivas (atenção e reflexividade), trabalhando com uma amostra de 98 crianças de 6 anos, consideradas em risco por pobreza.A pesquisa-ação, com delineamento pré-teste/intervenção/pós-teste, contou com a utilização de grupo controle (crianças sem risco) como parâmetro de comparação apenas no pré-teste.

A intervenção foi realizada em sala de aula pelos pesquisadores, em períodos de 30 minutos, três a quatro vezes por semana e durante um ano, sendo que os professores foram treinados para realizar atividades semelhantes em outros períodos. Como medidas de resultados, foram utilizados testes padronizados para avaliar a capacidade de reflexividade por oposição à impulsividade e a atenção seletiva. Uma tarefa de escrita de palavras foi utilizada para avaliar as habilidades de CF (considerada como a capacidade não só de isolar os fonemas, mas também de associá-los aos grafemas correspondentes). O Grupo Experimental apresentou melhoras significativas no pós-teste, tanto nas habilidades linguísticas, quanto nas cognitivas.

A pesquisa de Valenzuela et al. (2011), por sua vez, analisou os efeitos de um programa psicoeducativo aplicado a crianças identificadas pelas professoras como tendo baixo rendimento em leitura e escrita, além de terem tido baixo desempenho nas provas padronizadas de exatidão e compreensão leitora e de exatidão em cópia e ditado. A amostra ficou composta por 56 crianças de 5 anos (no início da pesquisa), as quais foram seguidas por três anos, configurando, assim, um estudo longitudinal com delineamento quase-experimental, com quatro testagens e três períodos de um ano de intervenção.

O programa, voltado para a promoção das habilidades fonológicas, semânticas e morfossintáticas, além da leitura e escrita, foi elaborado com a participação das professoras, que também receberam treinamento e supervisão para a sua aplicação, ocorrida por duas horas e meia por dia, durante 20 semanas por ano. Os resultados mostram diferenças significativas a favor do grupo experimental em leitura e escrita em todos os pós-testes, enquanto no grupo controle identificaram-se ganhos significativos apenas nas comparações da $4^{\mathrm{a}}$ avaliação com as $1^{\mathrm{a}}$ e $2^{\mathrm{a}}$ avaliações.

O último artigo desse grupo (Horta, 2012) relata uma pesquisa-ação de abordagem qualitativa, que buscou compreender como atividades de "escrita inventada" podem contribuir para a aprendizagem da escrita. Os participantes foram 38 crianças de 5 anos, que, no início da pesquisa, encontravam-se no estágio pré-silábico da escrita. A 
intervenção ocorreu em quatro sessões individuais de 15 minutos, baseadas na escrita inventada de palavras ditadas pela pesquisadora, que suscitava reflexões confrontando a produção da criança com escritas de nível conceptual mais avançado. Antes da intervenção, foram obtidas medidas de $\mathrm{CF}$, conhecimento de letras e escrita inventada. Os resultados mostram evolução conceptual em $71 \%$ das crianças pesquisadas, tendo sido observadas correlações significativas entre fonetização da escrita e conhecimento de letras, mas não com CF.

\section{Programas Focados na Linguagem Oral / Leitura Compartilhada}

Os programas que tiveram como foco as habilidades de linguagem oral basearam-se, todos, na leitura compartilhada e dialógica de livros infantis como estratégia promotora das habilidades de letramento (Tabela 2). O artigo de González e Contreras (2009) reporta dados do programa "Mochila Mágica", utilizado oficialmente há dez anos em algumas pré-escolas públicas da Venezuela. O programa baseia-se no empréstimo de livros e na formação de professores e pais para o apoio a atividades de leitura compartilhada e na promoção de atividades enfatizando as funções sociais da escrita.

No artigo, os autores descrevem os resultados de um estudo-piloto de um ano, realizado nos moldes de uma pesquisa-ação de abordagem qualitativa, envolvendo 179 crianças, 21 professores e 80 pais. Os resultados, obtidos a partir de entrevistas com os participantes, destacam aspectos positivos no interesse e estratégias dos alunos frente à leitura, assim como na participação e boa aceitação dos pais. Os professores apresentaram mudanças de atitude em relação ao ensino da língua escrita, mas também certa resistência à execução do programa.

Os estudos de Verzolla, Isotani e Perissinoto (2012) e de Borzone (2005) analisaram os efeitos da leitura compartilhada sobre as habilidades de linguagem oral de pré-escolares. O estudo de Verzolla et al. (2012), realizado com 58 crianças com idade entre 5 e 6 anos, baseou-se num delineamento pré-experimental (pré-teste/intervenção/pósteste sem grupo controle), tendo como medida de resultado narrativas orais, produzidas a partir da visualização de cinco figuras compondo uma estória. A intervenção foi realizada pela pesquisadora, nas salas de aula, com grupos de 20 a 30 alunos, consistindo em 10 sessões semanais de 30 minutos, nas quais ocorria a leitura compartilhada de um livro por sessão.Os resultados indicam aumento significativo nas habilidades narrativas de eventos centrais.

Já o estudo de Borzone (2005), caracterizado pela autora como uma pesquisa piloto de caráter exploratório, utilizou um delineamento com grupos de comparação e análise descritiva dos resultados e se propôs a analisar a influência da leitura compartilhada de contos infantis sobre as habilidades de compreensão e expressão de narrativas em crianças de grupos marginalizados. As medidas de desempenho foram obtidas solicitando-se um relato (narrativa) com base em um livro de figuras (do inglês, picture books), tarefa que combina habilidades de compreensão e produção oral.

Os participantes foram 39 crianças de 5 anos, divididas em três grupos: o grupo de intervenção (GI), com 14 crianças de NSE baixo de meio urbano, o grupo de comparação 1 (GC1), com 13 crianças de NSE baixo de meio rural e o grupo de comparação 2 (GC2), com 12 crianças de NSE médio. A professora foi treinada para realizar a leitura compartilhada de histórias três vezes por semana para o GI. No final do ano, essas crianças haviam desenvolvido sua capacidade narrativa, produzindo histórias bem estruturadas, apresentando desempenho muito superior ao $\mathrm{GC1}$, equiparando-se e, sob alguns aspectos, até mesmo superando o GC2.

O estudo de Fontes e Cardoso-Martins (2004) avaliou os efeitos da leitura dialógica e compartilhada de histórias sobre a compreensão, o vocabulário e as habilidades de escrita de pré-escolares. A pesquisa utilizou um delineamento experimental com grupo controle e foi realizada com uma amostra de 38 crianças de NSE baixo, com idades entre $4 \mathrm{e}$ 6 anos. a intervenção consistiu em 16 sessões semanais de leitura interativa de histórias, com duração de 30 minutos, realizada pela pesquisadora, com grupos de duas a quatro crianças. Para avaliar os resultados, foram usadas provas não padronizadas de conhecimento de letras, escrita e leitura de palavras e compreensão oral, além do subteste Vocabulário da Escala WISC. Os resultados indicam que as crianças do Grupo Experimental tiveram desempenho significativamente superior às do Grupo Controle apenas em compreensão e vocabulário.

\section{Programas Mistos}

O último grupo de estudos é formado por programas visando integrar e/ou comparar atividades voltadas para o desenvolvimento de habilidades focadas na compreensão do sistema alfabético e habilidades de linguagem oral (Tabela 3). O estudo de Marder (2011) investigou os efeitos de um programa de desenvolvimento de competências de letramento emergente sobre as habilidades de leitura de uma amostra de 35 pré-escolares, acompanhados dos 4 aos 6 anos de idade. A pesquisa utilizou delineamento constituído por pré-teste, intervenção, pós-teste 1, pós-teste 2 e pós-teste 3 (follow up), com grupo controle.

O programa, aplicado por professoras treinadas, decorreu durante dois anos, com uma periodicidade de duas a três horas semanais e compreendeu atividades voltadas para a promoção de conhecimentos sobre o sistema de escrita e da sua funcionalidade e atividades de leitura compartilhada de contos infantis, voltadas para a promoção da compreensão oral e do vocabulário. Para medir os resultados, foram usadas provas padronizadas de leitura de palavras e pseudopalavras e tarefa não padronizada de leitura de um pequeno 


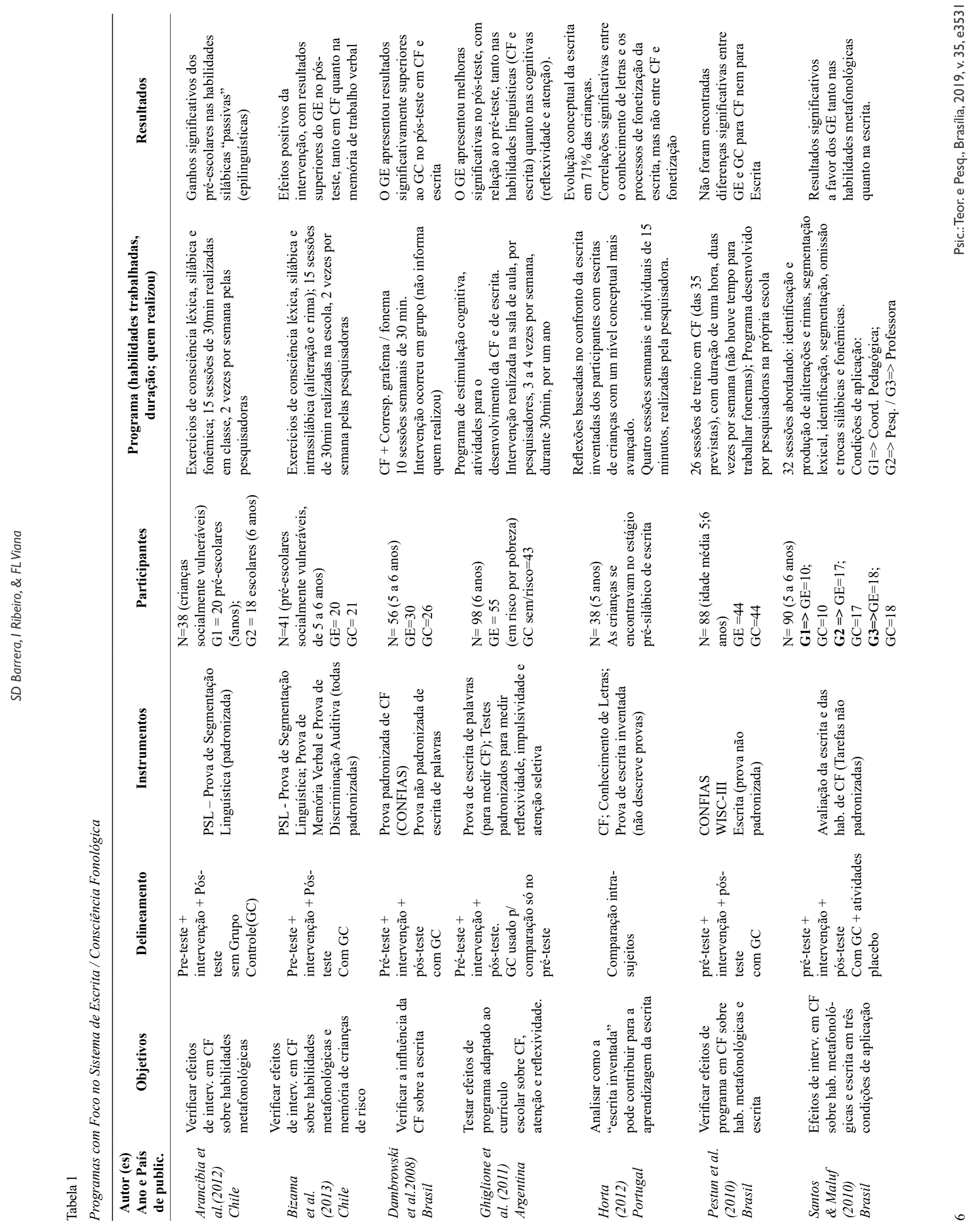



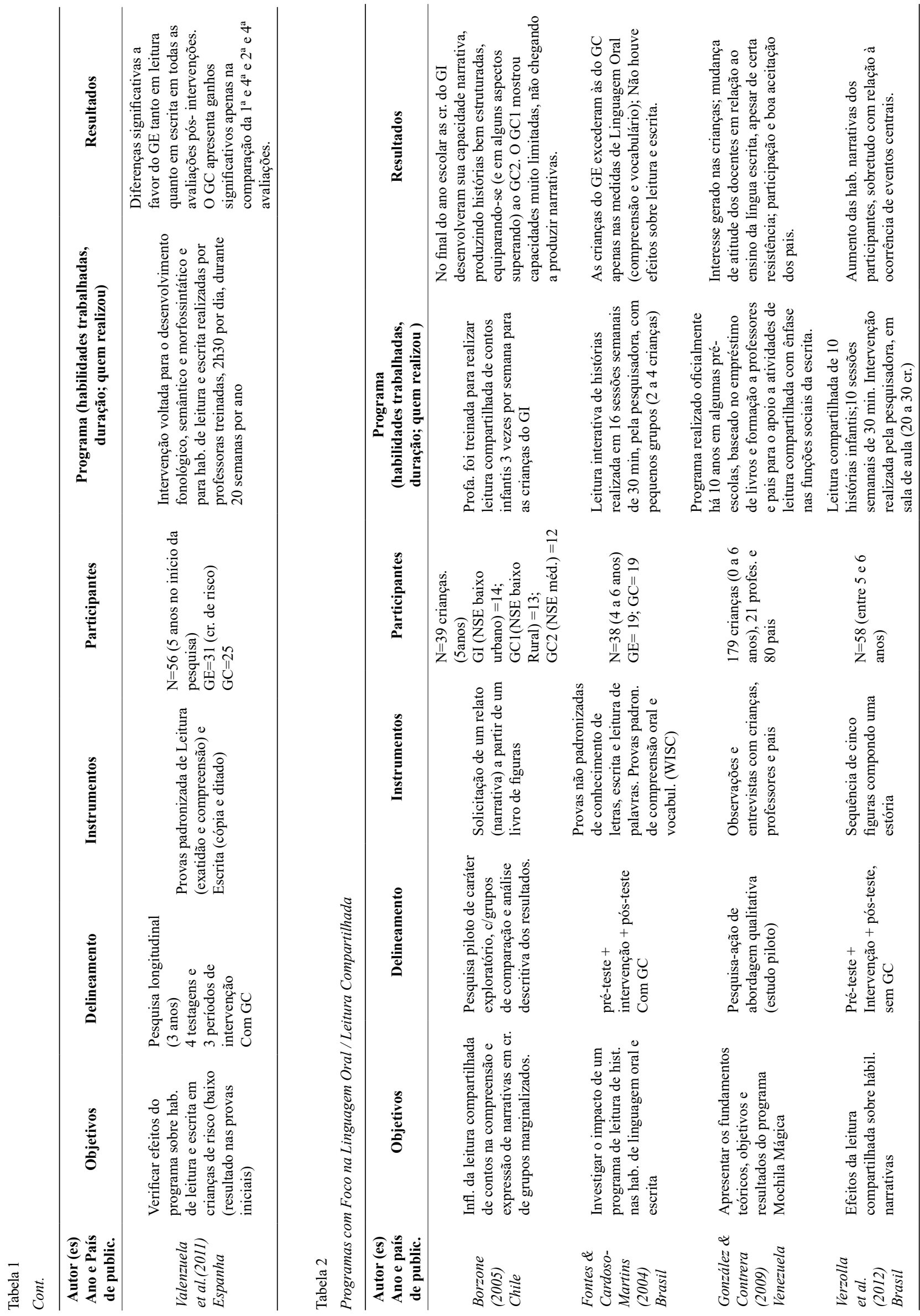

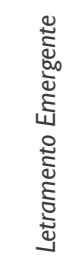
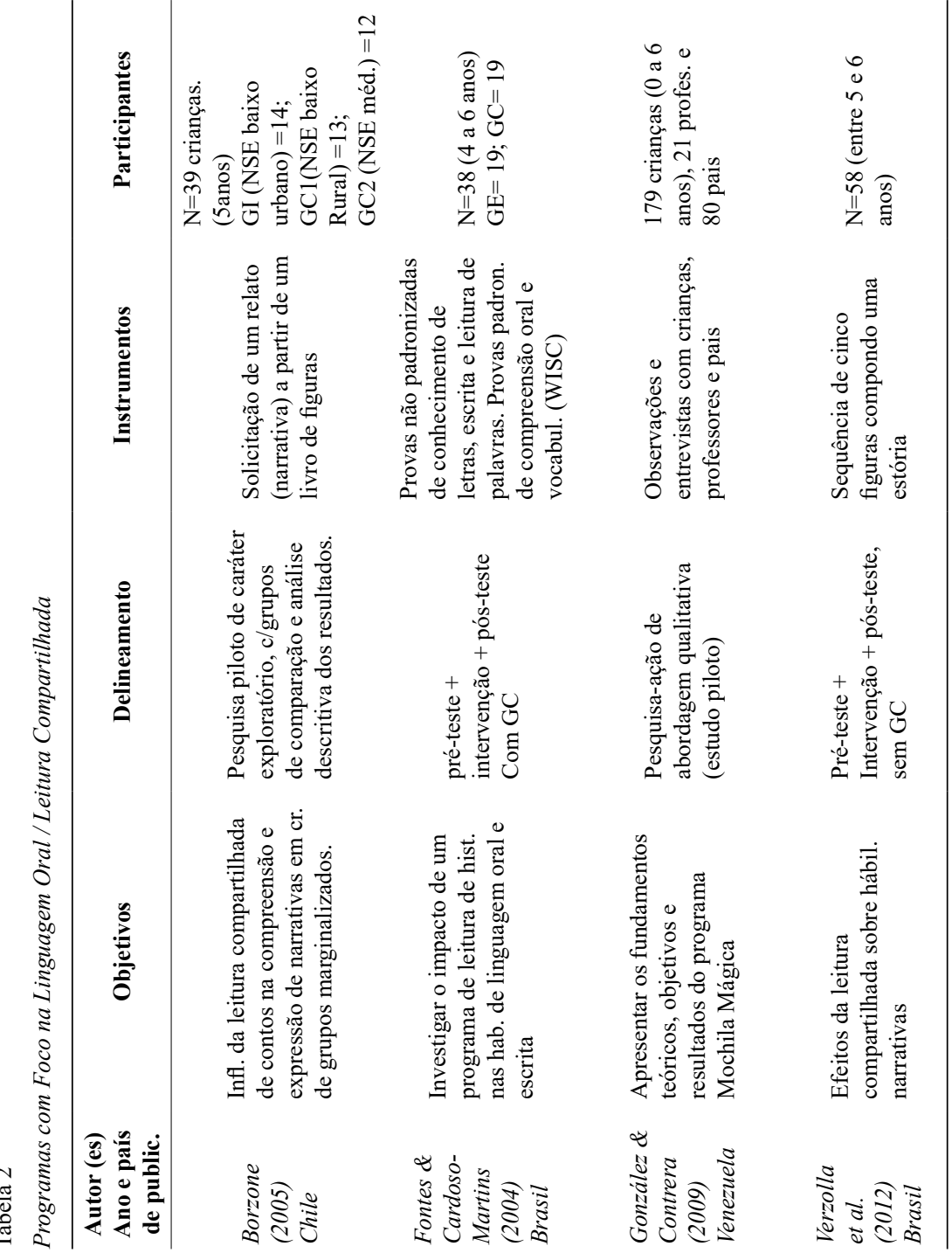

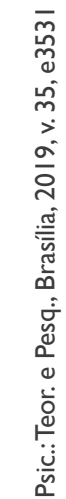




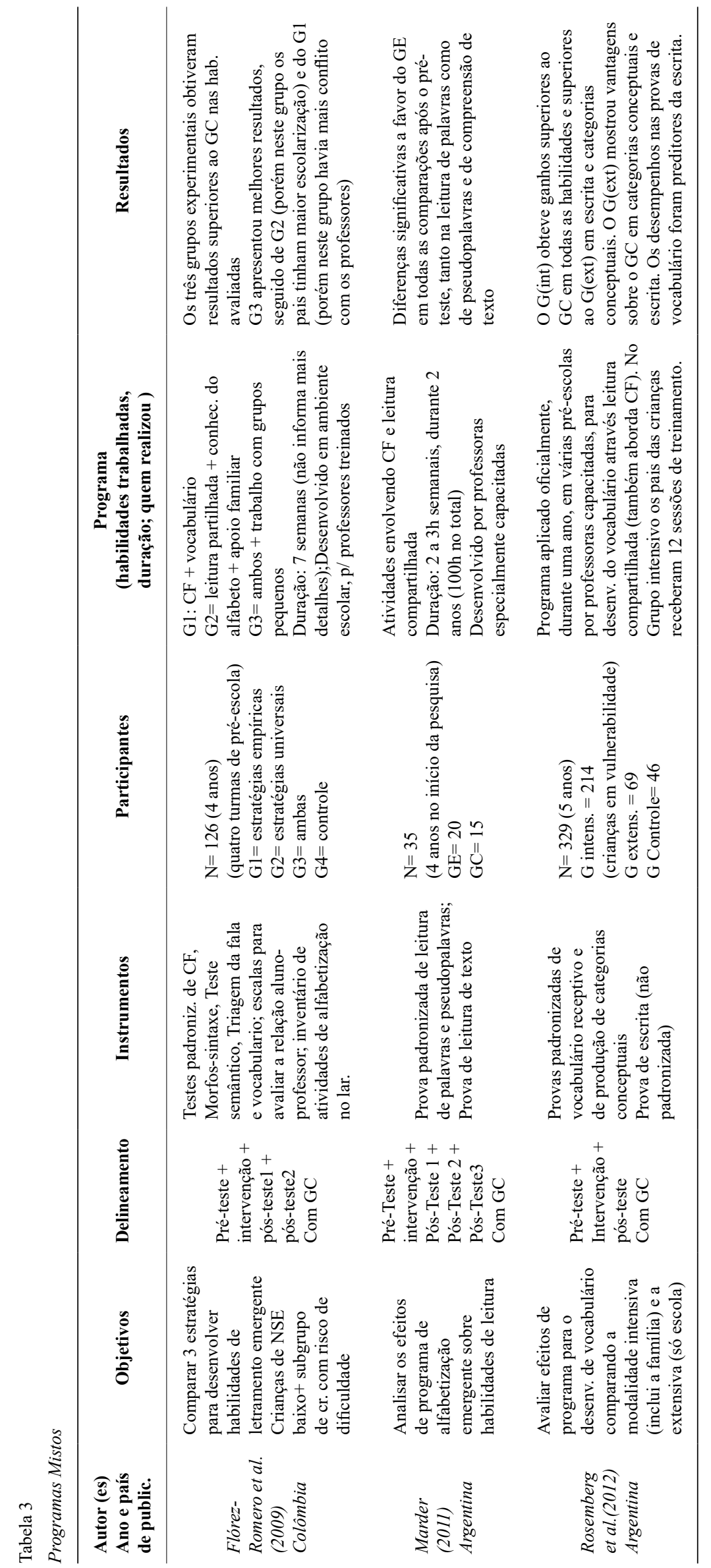

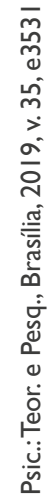

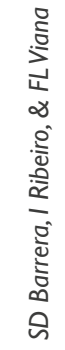


texto (aplicada quando os alunos estavam no $1^{\circ}$ ano). Os resultados mostraram diferenças significativas a favor do Grupo Experimental em todos os pós-testes e para todas as tarefas de leitura.

Rosemberg, Stein e Menti (2012) avaliaram os efeitos de um programa visando ao desenvolvimento do vocabulário, da aprendizagem de conceitos e de habilidades de escrita (incluindo CF), em uma amostra de 329 pré-escolares de NSE baixo, levando em conta variações na forma de aplicação. Os participantes foram divididos em três grupos: o primeiro, com 69 crianças, participou do programa apenas em contexto escolar, sendo considerado de "modalidade extensiva"; o segundo grupo, com 214 crianças em situação de vulnerabilidade, constituiu a "modalidade intensiva", com intervenção em contexto escolar e familiar, após os pais terem recebido 12 sessões de treinamento, visando à promoção das habilidades linguísticas e conceptuais em contexto de leitura compartilhada de contos; já o terceiro grupo, com 46 crianças, constituiu o controle.

Para avaliar os resultados, foram usadas provas padronizadas de vocabulário receptivo e de produção de categorias conceptuais, além de uma prova não padronizada de escrita de palavras. O programa foi aplicado durante um ano por professoras previamente treinadas. Como resultado, o grupo "modalidade intensiva" obteve os maiores ganhos em todas as habilidades, sendo essas diferenças significativas tanto em relação ao grupo modalidade extensiva, quanto ao grupo controle, com exceção da prova de vocabulário, em que a diferença é observada apenas em relação ao grupo controle. Os ganhos do grupo modalidade extensiva superaram significativamente os do grupo controle em desenvolvimento conceptual e em escrita. Além disso, os resultados em vocabulário mostraram-se preditores das habilidades de escrita.

O estudo de Flórez-Romero et al. (2009) comparou os efeitos de três estratégias para o desenvolvimento do letramento emergente, utilizando um delineamento quase-experimental, incluindo pré-teste, intervenção, pós-teste 1 e pós-teste 2 (follow up), com recurso a um grupo controle. Os participantes foram 4 professores, 105 pais e 126 crianças de 4 anos, de NSE baixo, alunas de quatro turmas de pré-escola, sendo que a cada turma foi atribuída uma das seguintes condições: Grupo 1 - programa baseado no que os autores denominam "estratégias universais" de letramento emergente, ou seja, atividades de leitura compartilhada e voltadas para o conhecimento do alfabeto; Grupo 2 - programa baseado em "estratégias empíricas" de letramento emergente, isto é, atividades voltadas para o desenvolvimento da $\mathrm{CF}$ e do vocabulário; Grupo 3 - programa "misto", envolvendo ambos os tipos de atividades, ocorridas em pequenos grupos para crianças identificadas como em risco para apresentarem dificuldades de aprendizagem; Grupo 4, turma que realizou apenas as atividades escolares habituais.

Os programas foram aplicados por professoras treinadas, durante sete semanas. Como medidas de resultados, foram utilizadas provas padronizadas de CF, do conhecimento de letras, das habilidades morfossintáticas e linguísticas (teste semântico e triagem da fala). Um inventário de atividades de alfabetização no lar e escalas para avaliar a relação professor-aluno também foram utilizadas. Como resultado, os desempenhos dos grupos experimentais foram superiores aos do grupo controle, com o grupo misto apresentando os melhores desempenhos, seguido do grupo submetido às estratégias universais e, em terceiro lugar, o grupo submetido às estratégias empíricas. Os autores referem, porém, que, no grupo submetido às estratégias universais, os pais eram mais escolarizados, enquanto no grupo submetido às estratégias empíricas havia maior grau de conflito nas relações professor-aluno.

\section{DISCUSSÃO}

Os programas analisados diferem na atenção e ênfase conferidas às diferentes habilidades de letramento emergente e às estratégias utilizadas para promovê-las. $\mathrm{O}$ primeiro grupo de estudos, englobando praticamente a metade das pesquisas analisadas, diz respeito às intervenções voltadas para o desenvolvimento de habilidades mais diretamente relacionadas ao domínio do sistema alfabético de escrita, sendo que, a maior parte delas, abordou, de forma mais ou menos sistemática, o desenvolvimento da CF.

Chama a atenção o fato das pesquisas desse grupo terem priorizado o efeito das intervenções sobre as habilidades de escrita, sendo que apenas uma delas (Valenzuela et al., 2011) analisou também sua influência sobre a leitura, diferentemente do que ocorre nas publicações de língua inglesa, que costumam priorizar as relações entre $\mathrm{CF}$ e leitura (Bus \& van IJzendoorn, 1999; Ehri, Nunes, Willow, Schuster, \& Yaghoub-Zadeh, 2001). Uma hipótese para a explicação de tal fato diz respeito à grande disseminação da teoria de Ferreiro e Teberosky (1986) - a qual descreve etapas psicogenéticas para a aquisição da escrita alfabética -, na América Latina e demais países de língua portuguesa e espanhola.

Quanto à eficácia dos programas analisados, constatou-se que apenas um (Pestun et al., 2010) não obteve resultados positivos, quer com relação à $\mathrm{CF}$, quer com relação à escrita. As autoras discutem o fato informando que não houve tempo para trabalhar a consciência fonêmica, referindo, ainda, o número elevado de crianças por turma, assim como certa inexperiência da pesquisadora que aplicou o programa, fatores esses que podem ter diminuído a eficácia do mesmo. 
Os resultados positivos dos outros sete estudos oferecem apoio a dados da literatura, que apontam a efetividade da intervenção em CF para o incremento dessas habilidades e também das habilidades de leitura e escrita, conforme indicam estudos de meta-análise realizados (Bus \& van IJzendoorn, 1999; Ehri et al., 2001).

A análise metodológica desse grupo de oito estudos indica que cinco deles seguiram um delineamento mais rigoroso (Bizama et al., 2013, Dambrowski et al., 2008; Pestun et al., 2010; Santos \& Maluf, 2010; Valenzuela et al., 2011). A pesquisa de Arancibia et al. (2012) não utilizou grupo controle, enquanto no estudo de Ghiglione et al. (2011), este foi usado como parâmetro apenas no pré-teste. Já a pesquisa de Horta (2012) utilizou comparação intrasujeitos. Embora apenas no estudo de Santos e Maluf(2010) o grupo controle tenha participado de atividades placebo a fim de controlar um possível Efeito Hawthorne, salienta-se que tal efeito não foi encontrado na meta-análise realizada por Ehri et al. (2001).

A análise dos instrumentos utilizados nas pesquisas indica que cinco usaram testes padronizados para medir a CF (Arancibia et al., 2012; Bizama et al., 2013; Dambrowski et al., 2008; Ghiglione et al., 2011; Pestun et al., 2010), uma utilizou tarefa de CF elaborada pelas pesquisadoras (Santos \& Maluf, 2010), outra não descreveu o instrumento usado (Horta, 2012) e uma não avaliou essa habilidade, embora o programa contemplasse o seu desenvolvimento (Valenzuela et al., 2011). Quanto à avaliação da escrita, realizada em seis estudos desse grupo, apenas Valenzuela et al. (2011) utilizaram testes padronizados, o que sugere certa carência de provas padronizadas para avaliação das habilidades iniciais de escrita em português e em espanhol.

É importante analisar também aspectos relacionados às condições de aplicação dos programas, as quais têm sido consideradas como fatores moderadores capazes de influenciar os resultados da intervenção (Piasta \& Wagner, 2010), além de serem importantes no sentido de se pensar na eficiência dos programas para além de sua eficácia. Considerando o número de crianças que efetivamente participaram das intervenções, este variou de 20 a 56 alunos, sendo que, na grande maioria dos estudos, as atividades instrucionais ocorreram coletivamente, em contexto escolar, porém tendo sido aplicadas pelas equipes de pesquisa. As exceções foram o estudo de Horta (2012), com intervenção individual, o estudo de Valenzuela et al. (2011), no qual a intervenção foi efetuada por professoras treinadas e a pesquisa de Santos e Maluf (2010), na qual três turmas de pré-escolares foram submetidas a intervenção por diferentes agentes (professora, pesquisadora e coordenadora pedagógica), não encontrando diferenças significativas entre as condições de aplicação. Tais resultados demonstram a potencialidade dos programas para serem aplicados em situação de sala de aula, sugerindo a viabilidade dos mesmos serem utilizados pelos professores, desde que tenham recebido a formação adequada para o seu desenvolvimento.
Por fim, atenção especial deve ser dada à estrutura e conteúdo dos programas analisados. A maioria dos estudos fornece informações gerais sobre as atividades e materiais utilizados e/ou remete o leitor para a literatura na qual o programa se baseia. Dois artigos (Horta, 2012; Valenzuela et al., 2011) oferecem informações mais detalhadas, no primeiro caso sobre os procedimentos e, no segundo, sobre os conteúdos trabalhados. Dos cinco estudos que se focaram prioritariamente no desenvolvimento da CF (Bizama et al., 2013; Arancibia et al., 2012; Dambrowski et al., 2008; Pestun et al., 2010; Santos \& Maluf, 2010), a natureza dos programas parece bastante semelhante, iniciando com atividades voltadas à análise e manipulação dos segmentos mais amplos da fala, até chegar a atividades de manipulação de fonemas, embora dois estudos não tenham chegado a trabalhar ou enfatizar essa última parte com as amostras de pré-escolares (Arancibia et al., 2012; Pestun et al., 2010).

É importante lembrar que o programa desenvolvido por Dambrowski et al. (2008) trabalhou as correspondências entre letras e sons, sendo que outros três estudos também trabalharam essa habilidade, enfocando, ainda, atividades mais específicas de escrita, como foi o caso dos programas desenvolvidos por Ghiglione et al. (2011), Valenzuela et al. (2011) e Horta (2012). Tais propostas estão de acordo com o consenso observado na literatura de que maiores efeitos sobre a leitura e escrita são obtidos a partir de intervenções em CF, que abordam também as correspondências entre fonemas e grafemas (Bus \& van IJzendoorn, 1999; Bryant \& Bradley, 1987; Ehri et al., 2001; Hatcher et al., 1994).

A análise da frequência e duração dos programas indica que aqueles que focaram mais especificamente o desenvolvimento da CF desenvolveram intervenções que variaram de 5 horas (Dambrowski et al., 2008) a 26 horas (Pestun et al., 2010), sendo esse último o único que não obteve efeitos estatisticamente significativos. Tais resultados vão ao encontro daqueles obtidos na meta-análise empreendida por Ehri et al. (2001), os quais indicam que os programas visando ao desenvolvimento da $\mathrm{CF}$ e das habilidades de leitura e escrita que se mostraram mais eficazes foram aqueles cuja duração variou entre 5 e 18 horas, sendo que intervenções mais curtas ou mais longas mostraram-se estatisticamente menos eficazes.

Entretanto, os programas realizados por Valenzuela et al. (2011) e Ghiglione et al. (2011), apesar da duração bem mais longa, foram também eficazes, o que pode ser explicado por estarem integrados nas rotinas pedagógicas, o primeiro com atividades diárias durante três anos e o segundo com atividades três a quatro vezes por semana, durante um ano, abordando, além da CF, atividades de escrita. Já a intervenção realizada por Horta (2012), efetuada em apenas quatro sessões de 15 minutos, pode ter sido potencializada pelo fato de ter ocorrido de forma individual.

Com relação ao segundo grupo de programas analisados, observa-se que o seu denominador comum reside no fato de que os quatro estudos se basearam na estratégia de 
leitura compartilhada e dialógica de livros infantis para o desenvolvimento de habilidades de letramento emergente. Três desses estudos tiveram como objetivo testar os efeitos dessa estratégia sobre habilidades linguísticas, como vocabulário (Fontes \& Cardoso-Martins, 2004), compreensão oral (Borzone, 2005) e habilidades narrativas (Borzone, 2005; Verzolla et al., 2012), tendo os mesmos obtido resultados positivos, o que vai ao encontro dos dados da literatura (Lever \& Sénéchal, 2011; Mol et al., 2008; Wasik et al., 2006).

Desses programas, um foi aplicado por professora treinada para realizar a leitura dialógica (Borzone, 2005), o que remete à possível utilização dessa estratégia como prática pedagógica regular. O estudo de Fontes e CardosoMartins (2004) é relevante também, na medida em que não encontrou efeitos positivos da leitura compartilhada sobre as habilidades de escrita. Entretanto, resultados obtidos por Justice et al. (2010) sugerem que práticas de leitura compartilhada podem ter efeitos positivos nos conhecimentos de pré-escolares sobre a escrita, desde que essa leitura seja acompanhada por estratégias sistemáticas e intencionais, visando atrair a atenção das crianças para aspectos específicos da linguagem escrita, tais como: letras, palavras, sinais de pontuação e direção da leitura.

Já o estudo de González e Contrera (2009), por sua vez, descreve um programa oficial baseado no empréstimo de livros e na formação a professores e pais para o apoio a atividades de leitura compartilhada dialógica, sem especificar os efeitos do mesmo sobre habilidades linguísticas em particular. Com efeito, a abordagem teórica do letramento emergente tem considerado o ambiente familiar como um contexto privilegiado para o desenvolvimento de conhecimentos e habilidades relacionadas à aprendizagem da leitura e da escrita, através da participação da criança em práticas interativas que tenham como objeto a linguagem escrita (Whitehurst \& Lonigan, 1998).

A leitura de histórias infantis pelos adultos vem sendo considerada uma prática importante no desenvolvimento das habilidades de letramento emergente, não apenas por sua potencialidade para a promoção de habilidades linguísticas, mas também pela motivação que provoca em crianças de diferentes idades (Mol et al., 2008). Entretanto, estudos têm demonstrado que esse padrão compartilhado de leitura não ocorre com a mesma frequência e qualidade interativa nos lares das crianças de NSE mais baixo, as quais, em geral, contam também com pais menos escolarizados (Levin \& Aram, 2010). Assim, justifica-se a importância de pesquisas dessa natureza, sugerindo mais uma forma de favorecer as competências de letramento emergente a todas as crianças.

Dentre os programas desse segundo grupo, o estudo de Fontes e Cardoso-Martins (2004) foi o que apresentou delineamento mais rigoroso, utilizando não apenas comparações entre pré e pós-teste, mas também com grupo controle. O referido estudo foi também o único que utilizou medidas padronizadas para avaliar as habilidades linguísticas - no caso o vocabulário. Tal fato aponta limitações importantes na maior parte dos estudos de intervenção em letramento emergente, visando ao desenvolvimento da linguagem oral na literatura ibero-americana, sugerindo a necessidade de maior rigor metodológico em futuras pesquisas, de modo a aumentar a confiabilidade dos resultados obtidos.

No último grupo de estudos analisados, encontramos programas como os de Marder (2011) e Rosemberg et al. (2012), que apresentam uma proposta mais abrangente, visando integrar o desenvolvimento de diferentes tipos de habilidades relacionadas ao letramento emergente, tais como vocabulário, $\mathrm{CF}$ e mesmo habilidades conceptuais, no caso do segundo estudo. Esse buscou, ainda, comparar os efeitos do programa aplicado apenas em ambiente escolar com uma modalidade intensiva, na qual os pais das crianças também foram treinados para o desenvolvimento de leitura compartilhada de histórias e para exploração de rimas e aliterações quando da leitura das mesmas.

Ambos os estudos utilizaram programas previamente elaborados para serem aplicados em contexto escolar, sendo os mesmos aplicados por professores especialmente capacitados, tendo obtido resultados bastante positivos sobre habilidades linguísticas (conceptuais), linguísticas, metalinguísticas e também sobre a leitura. Já no estudo de Flórez-Romero et al. (2009), que comparou os efeitos de três programas visando desenvolver as habilidades de letramento emergente a partir de diferentes estratégias metodológicas, as atividades também foram aplicadas por professoras treinadas, sendo que o grupo que recebeu o programa integrando habilidades linguísticas e metalinguísticas foi o que obteve os melhores resultados.

Este estudo teve o mérito de incluir algumas variáveis que podem interferir nos resultados, como a relação professor-aluno e o grau de instrução dos pais, porém, como limitação, apresenta o fato de não ter realizado nenhuma medida específica de leitura ou de escrita. Uma característica importante desse último grupo de estudos diz respeito ao fato de que todos eles, embora apresentando delineamento experimental ou quase-experimental, aproximam-se mais de contextos naturais, tanto no que se refere ao ambiente de execução dos programas, quanto aos aplicadores, apresentando, portanto, maior validade externa. Além disso, utilizaram provas padronizadas para avaliar as habilidades linguísticas (vocabulário) e de leitura, o que aumenta a confiabilidade dos resultados obtidos. 


\section{CONSIDERAÇÕES FINAIS}

Apesar da diversidade dos estudos analisados quanto à metodologia utilizada e quanto aos objetivos, conteúdos e estratégias dos programas desenvolvidos, apenas um deles não obteve efeitos positivos sobre as habilidades de letramento emergente e/ou de linguagem escrita, o que é encorajador. Além disso, tanto os programas focados nos conhecimentos e habilidades relacionados ao código escrito, quanto aqueles focados no desenvolvimento da linguagem oral mostraram resultados positivos, sendo que aqueles integrando ambos os tipos de conhecimentos e habilidades parecem ter tido maior efeito sobre as competências de letramento emergente.

Entretanto, questões metodológicas observadas em parte dos estudos analisados, os quais não empregaram grupo de comparação para controle de possíveis variáveis intervenientes, bem como utilizaram provas não padronizadas para avaliar o desempenho dos participantes, sugerem certa cautela quanto ao grau de confiabilidade dos resultados obtidos. Tal ressalva já havia sido feita por Shanahan e Lonigan (2010) a respeito dos estudos analisados para a elaboração do NELP e permanece válida para a atual revisão.

Os artigos analisados, em geral, apresentam boa fundamentação teórica a respeito das habilidades de letramento emergente e da sua relação com a aprendizagem da leitura e da escrita. Porém, um aspecto que limita a qualidade de parte dos estudos diz respeito à falta de uma apresentação mais sistemática e detalhada, não apenas da estrutura dos programas implementados em relação à duração, frequência e condições de aplicação, mas também quanto aos conteúdos trabalhados, o que dificulta a replicação das intervenções efetuadas.

$\mathrm{O}$ fato de vários programas terem sido aplicados em contexto natural, por professores e como parte das atividades escolares, se por um lado diminui o controle de algumas variáveis, limitando a validade interna dos estudos, por outro, nos permite antever sua possível aplicabilidade em situações reais, aumentando, assim, sua validade externa. Uma lacuna a ser preenchida, entretanto, refere-se à falta de estudos com amostras maiores, que permitam avaliar a real efetividade dos programas implementados.

Embora dirigidos a grupos de alunos considerados em vulnerabilidade para apresentar futuras dificuldades na escolarização, os programas analisados parecem contemplar aspectos importantes a serem trabalhados na educação pré-escolar de modo geral, sobretudo de forma integrada e contextualizada, a fim de favorecer o desenvolvimento de habilidades cognitivas e linguísticas que têm se mostrado favorecedoras da aprendizagem da leitura e da escrita.

\section{REFERÊNCIAS}

Arancibia, B., Bizama, M., \& Sáez, K. (2012). Aplicación de un programa de estimulación de la conciencia fonológica en preescolares de nivel transición 2 y alumnos de primer año básico pertenecientes a escuelas vulnerables de la Provincia de Concepción, Chile. Revista Signos, 45(80), 236-256.

Bizama, M., Arancibia, B., \& Sáez, K. (2013). Intervención psicopedagógica temprana en conciencia fonológica como proceso metalingüístico a la base de la lectura en niños de 5 a 6 años socialmente vulnerables. Estudios Pedagógicos, 2, 25-39.

Borzone, A. M. (2005). La lectura de cuentos en el Jardín Infantil : Un medio para el desarrollo de estrategias cognitivas y lingüísticas. Psykhe (Santiago), 14(1), 192-209.

Bryant, P., \& Bradley, L. (1987). Problemas de leitura na criança. Porto Alegre: Artes Médicas.

Bus, A. G., \& van IJzendoorn, M. H. (1999). Phonological awareness and early reading: A meta-analysis of experimental training studies. Journal of Educational Psychology, 91(3), 303-414.

Campos, M. M., Bhering, E. B., Esposito, Y., Gimenes, N., Abuchaim, B., Valle, R., \& Unbehaum, S. (2011). A contribuição da educação infantil de qualidade e seus impactos no início do ensino fundamental. Educação e Pesquisa, 37(1), $15-33$.

Capovilla, A. G. S., \& Capovilla, F. C. (2000). Efeitos do treino de consciência fonológica em crianças com baixo nível sócioeconômico. Psicologia: Reflexão e Crítica, 13(1), 7-24.

Capovilla, A. G. S., Capovilla, F. C., \& Machalous, N. (2005). Avaliação das estratégias de leitura fonológica e lexical em português e alemão: Efeito da ortografia. Boletim de Psicologia, 55(122), 59-82.
Cardoso-Martins, C., \& Batista, A. C. E. (2005). O conhecimento do nome das letras e o desenvolvimento da escrita: Evidência de crianças falantes do português. Psicologia: Reflexão $e$ Crítica, 18(3), 330-336.

Dambrowski, A. B., Martins, C. L., Theodoro, J. D. L., \& Gomes, E. (2008). Influência da consciência fonológica na escrita de pré-escolares. Revista CEFAC, 10(2), 175-181.

Dickinson, D. K., McCabe, A., \& Essex, M. J. (2006). A window of opportunity we must open to all: The case of preschool with high-quality support for language and literacy. In D. K. Dickinson \& S. B. Neuman (Eds.), Handbook of Early Literacy (vol. 2, pp. 11-28). New York: Guilford Press.

Ehri, L. C., Nunes, S. R., Willow, D. M., Schuster, B. V., \& Yaghoub-Zadeh, Z. (2001). Phonemic awareness instruction helps children learn to read: Evidence from the National Reading Panel's meta-analysis. Reading Research Quarterly, $36(3), 250-287$.

Ellis, A. (2001). Leitura, escrita e dislexia: Uma análise cognitiva. Porto Alegre: Artmed.

Ferreiro, E., \& Teberosky, A. (1986). Psicogênese da língua escrita. Porto Alegre: Artes Médicas.

Flórez-Romero, R., Restrepo, M. A., \& Schwanenflugel, P. (2009). Promoción del alfabetismo inicial y prevención de las dificultades en la lectura: Una experiencia pedagógica en el aula de preescolar. Avances en Psicologia Latinoamericana, 27(1),79-96.

Fontes, M. J. O., \& Cardoso-Martins, C. (2004). Efeitos da leitura de histórias no desenvolvimento da linguagem de crianças de nível socioeconômico baixo. Psicologia: Reflexão e Crítica, 17(1), 83-94. 
Ghiglione, M. E., Filipetti, V., Manucci, V., \& Apaz, A. (2011). Programa de intervención, para fortalecer funciones cognitivas y linguísticas, adaptado al currículo escolar en niños en riesgo por pobreza. Interdisciplinaria, 28(1), 17-36.

Gombert, J. E. (2003). Atividades metalinguísticas e aquisição da leitura. In M. R. Maluf (Org.), Metalinguagem e aquisição da escrita (pp. 19-64). São Paulo: Casa do Psicólogo.

González, Z. M., \& Contreras, D. G. (2009). Transitando el camino de construcción de la lengua escrita, con La Mochila Mágica. Revista Latinoamericana Ciencias Sociales Niñez y Juventud, 7(2), 971-988.

Hatcher, P. J., Hulme, C., \& Ellis, A. W. (1994). Ameliorating early reading failure by integrating the teaching of reading and phonological skills the phonological linkage hypothesis. Child Development, 65(1), 41-57.

Horta, I. D. V. (2012). Da escrita grafo-perceptiva à escrita fonetizada: Factores e processos evolutivos na descoberta do princípio alfabético. Análise Psicológica, 4, 435-449.

Juel, C. (2006). The impact of early school experiences on initial reading. In D. K. Dickinson \& S. B. Neuman (Eds.), Handbook of early literacy (pp. 410-426). New York: Guilford Press.

Justice, L. M., McGinty, A.S., Piasta, S.B., Kaderavek, J.N., \& Fan, X. (2010). Print-focused read-alouds in preschool classrooms: Intervention effectiveness and moderators of child outcomes. Language, Speech and Hearing Services in Schools, 41, 504-520.

Lever, R., \& Sénéchal, M. (2011). Discussing stories: On how a dialogic reading intervention improves kindergartners' oral narrative construction. Journal of Experimental Child Psychology, 108(1), 1-24.

Levin, I., \& Aram, D. (2010). Mother-child joint writing and storybook reading and their effects on kindergartners' literacy: An intervention study. Reading and Writing, 25(1), 217-249.

Lonigan, C. J., Burgess, S. R., \& Anthony, J. L. (2000). Development of emergent literacy and early reading skills in preschool children: Evidence from a latent-variable longitudinal study. Developmental Psychology, 36(5), 596-613.

Marder, S. (2011). Resultados de un programa de alfabetización temprana. Desempeño en lectura en niños de sectores en desventaja socioeconomica. Interdisciplinaria, 28(1), 159-176

Melby-Lervag, M., Lyster, S. A. H., \& Hulme, C. (2012). Phonological skills and their role in learning to read: A metaanalytic review. Psychological Bulletin, 138 (2), 322-352.

Mol, S. E., Bus, A. G., de Jong, M. T., \& Smeets, D. J. H. (2008). Added value of dialogic parent-child book readings: A metaanalysis. Early Education \& Development, 19(1), 7-26.

Neuman, S. B., \& Dickinson, D. K. (2003). Introduction. In Handbook of Early Literacy (vol. 1, pp. 1-10). New York: Guilford Press.
Pestun, M. S. V., Omote, L. C. F., Barreto, D. C. M., \& Matsuo, T. (2010). Estimulação da consciência fonológica na educação infantil: Prevenção de dificuldades na escrita. Psicologia Escolar e Educacional, 14(1), 95-104.

Piasta, S. B., \& Wagner, R. K. (2010). Developing early literacy skills: A meta-analysis of alphabet learning and instruction. Reading Research Quarterly, 45(1), 8-38.

Rosemberg, C. R., Stein, A., \& Menti, A. (2012). Orientación educativa sobre el vocabulario y el acceso a la alfabetización. Evaluación del impacto de un programa de intervención en las familias y la escuela. Orientación y Sociedad, 11. Retrieved from http://www.scielo.org.ar/pdf/orisoc/v11/v11a03.pdf

Rosenshine, B. (2001). Issues in conducting meta-analysis of intervention studies. The Elementary School Journal, 101(3), 371-377.

Santos, M. J. dos, \& Maluf, M. R. (2010). Consciência fonológica e linguagem escrita: efeitos de um programa de intervenção. Educar em Revista, 38, 57-71.

Schatschneider, C., Fletcher, J. M., Francis, D. J., Carlson, C. D., \& Foorman, B. R. (2004). Kindergarten prediction of reading skills: A longitudinal comparative analysis. Journal of Educational Psychology, 96(2), 265-282.

Shanahan, T., \& Lonigan, C.J. (2010). The National Early Literacy Panel: A summary of the process and the report. Educational Researcher, 39(4), 279-285.

Storch, S. A., \& Whitehurst, G. (2002). Oral language and coderelated precursors to reading: Evidence from a longitudinal structural model. Developmental Psychology, 38(6), 934-947.

Torgesen, J. (2002). The prevention of reading difficulties. Journal of School Psychology, 40(1), 7-26.

Valenzuela, M. J. G., Ruiz, I. M., \& Ríos, M. D. (2011). Intervención temprana de la lecto-escritura en sujetos con dificultades de aprendizaje. Revista Latinoamericana de Psicologia,43(1), $35-44$

Verzolla, B. L. P., Isotani, S. M., \& Perissinoto, J. (2012). Análise da narrativa oral de pré-escolares antes e após estimulação de linguagem. Jornal da Sociedade Brasileira de Fonoaudiologia, 24(1), 62-68.

Wagner, R., \& Torgesen, J. (1987). The nature of phonological processing and its causal role in the acquisition of reading skills. Psychological Bulletin, 101,192-212.

Wasik, B. A., Bond, M. A., \& Hindman, A. (2006). The effects of a language and literacy intervention on Head Start children and teachers. Journal of Educational Psychology, 98(1), 63-74.

Whitehurst, G. J., \& Lonigan, C. J. (1998). Child development and emergent literacy. Child Development, 69(3), 848-72.

Whitehurst, G. J., \& Lonigan, C. J. (2003). Emergent literacy: development from pre-readers to readers. In S. B. Neuman \& D. K. Dickinson (Eds.), Handbook of early literacy (vol. 1, pp. 11-29). New York: Guilford Press. 